\title{
ANOTHER INVOLUTION ON MODULI OF SEXTICS
}

\author{
Mutsuo OKa
}

\section{Introduction}

Let $\mathscr{M}$ be the moduli space of sextics with 6 cusps and 3 nodes. A sextic $C$ is called of a $(2,3)$-torus type (or briefly of a torus type) if its defining polynomial $f$ has the expression $f(x, y)=f_{2}(x, y)^{3}+f_{3}(x, y)^{2}$ for some polynomials $f_{2}, f_{3}$ of degree 2, 3 respectively. We denote by $\mathscr{M}_{\text {torus }}$ the component of $\mathscr{M}$ which consists of curves of a torus type and by $\mathscr{M}_{\text {gen }}$ the curves of a general type (= not of a torus type). We denote the dual curve of $C$ by $C^{*}$. Recall that $C^{*}$ is the image of the Gauss map dual $C: C \rightarrow \boldsymbol{P}^{2 *},(X, Y, Z) \mapsto\left(F_{X}(X, Y, Z)\right.$, $\left.F_{Y}(X, Y, Z), F_{Z}(X, Y, Z)\right)$. In our previous paper [O3], we have shown that the dual curve operation $C \mapsto C^{*}$ gives an involution on $\mathscr{M}$ and it preserves the type of the curve in $\mathscr{M}$, i.e., $C^{*} \in \mathscr{M}_{\text {torus }}$ if and only if $C \in \mathscr{M}_{\text {torus. }}$. Let $G:=\operatorname{PGL}(3, C)$. The quotient moduli spaces are by definition the quotient spaces of the moduli spaces by the action of $G$.

The purpose of this note is to show that there exists an involution $\bar{\imath}$ on $\mathscr{M} / G$ such that $\bar{l}$ is different from the dual curve operation and $\bar{l}$ preserves the types of the sextics (Theorem 2.4).

For the construction of $\bar{l}$, we consider the moduli space $\tilde{\mathscr{M}}$ of plane curves of degree 12 with 24 cusps and 24 nodes. This moduli space is also self-dual in the sense that $C^{*} \in \tilde{\mathscr{M}}$ if $C \in \tilde{\mathscr{M}}$. The construction of $\bar{l}$ is done as follows. First observe that $C$ has three bi-tangent lines for any $C \in \mathscr{M}$. We take $g \in G$ so that the three coordinate lines $X=0, Y=0, Z=0$ are the bi-tangent lines of $C^{g}$ and let $F(X, Y, Z)=0$ be the defining homogeneous polynomial of degree 6 . Then consider the curve $\tilde{C}$ defined by $F\left(X^{2}, Y^{2}, Z^{2}\right)=0$. It turns out that $\tilde{C}$ is contained in $\tilde{\mathscr{M}}$. This operation defines a rational mapping $\psi: \mathscr{M} / G \rightarrow \tilde{\mathscr{M}} / G$. We define $\bar{\imath}(C)=\psi^{-1}\left(\psi\left(C^{g}\right)^{*}\right)$.

\section{Involution on the quotient moduli $\mathscr{M} / G$}

Let $\mathscr{M}$ and $\tilde{\mathscr{M}}$ be the moduli space of sextics with 6 cusps and three nodes and the moduli space of irreducible plane curves of degree 12 with 24 cusps and 24 nodes respectively. Note that the genus of a generic curve in $\mathscr{M}$ (respectively in $\tilde{\mathscr{M}})$ is 1 (resp. 7). By the class formula $([\mathrm{N}]$ or $[\mathrm{O} 3])$, it is easy to see that

Received December 7, 1999; revised June 7, 2000. 
for a generic $C \in \tilde{\mathscr{M}}$, the dual curve $C^{*}$ is also in $\tilde{\mathscr{M}}$. We consider the mapping $\pi: \boldsymbol{P}^{2} \rightarrow \boldsymbol{P}^{2}$, defined by $\pi(X, Y, Z)=\left(X^{2}, Y^{2}, Z^{2}\right)$, which is a 4-fold covering branched along the coordinate axes $\{X=0\} \cup\{Y=0\} \cup\{Z=0\}$. Take a generic curve $C \in \mathscr{M}$ and let $F(X, Y, Z)$ be the defining homogeneous polynomial of degree 6. As $C^{*}$ has three nodes, $C$ has three bi-tangent lines. We denote by $\mathscr{M}^{n m l}$ the subset of $\mathscr{M}$ which consists of curves $C \in \mathscr{M}$ whose three bi-tangent lines are $X=0, \quad Y=0$ and $Z=0$. We define a mapping $\psi: \mathscr{M}^{n m l} \rightarrow \tilde{\mathscr{M}}$ as follows. Let $C \in \mathscr{M}^{n m l}$ and let $F(X, Y, Z)$ be the defining homogeneous polynomial. We define $\psi(C):=\pi^{-1}(C)$. Note that $\psi(C)$ is defined by $\tilde{F}(X, Y, Z)$ $:=F\left(X^{2}, Y^{2}, Z^{2}\right)$. Each cusp of $C$ produces 4 cusps on $\psi(C)$. Thus $\psi(C)$ has 24 cusps. Each node of $C$ also gives 4 nodes on $\psi(C)$, thus we get 12 nodes on $\psi(C)$ which are mapped onto the nodes of $C$. As the restriction of $\pi$ to the affine chart $\{Z \neq 0\}$ is the composition of double coverings $(x, y) \mapsto\left(x, y^{2}\right)$ and $(x, y) \mapsto\left(x^{2}, y\right)$, each simple tangent on the coordinate axis $X=0, Y=0$ gives 2 nodes on $\psi(C)$ ([OO1]). This is the same for the simple tangents for $Z=0$. Thus there are 12 nodes on $\psi(C)$ which are on the three coordinate axes and they are mapped to simple tangents on coordinate axis by $\pi$. Thus $\psi(C)$ has 24 nodes. Thus $\psi(C) \in \tilde{\mathscr{M}}$.

Now for $C \in \mathscr{M}$, we define $\bar{\psi}(C)$ as $\psi\left(C^{g}\right)$ by choosing $g \in G$ such that $C^{g} \in$ $\mathscr{M}^{n m l}$. The ambiguity for the choice of $g \in G$ is in the stabilizer $G_{\mathscr{M}^{n m l}}$ of $\mathscr{M}^{n m l}$ which is a direct product of $\Xi_{3}$ (the permutations of coordinates) and $\boldsymbol{C}^{*} \times \boldsymbol{C}^{*} \times$ $C^{*}$ (scalar multiplications). Thus the polynomials $F(X, Y, Z)$ and $\tilde{F}(X, Y, Z)$ are unique up to a $G_{\mathscr{M}^{n m l}}$ action. Thus $\mathscr{M}^{n m l} / G_{\mathscr{M}^{n m l}} \cong \mathscr{M} / G$ and $\bar{\psi}: \mathscr{M} / G \rightarrow$ $\tilde{\mathscr{M}} / G$ is well-defined.

Recall that a polynomial $F(X, Y, Z)$ is called even in $X$ (respectively symmetric in $X, Y)$ if $F(-X, Y, Z)=F(X, Y, Z) \quad($ resp. $\quad F(Y, X, Z)=$ $F(X, Y, Z))$. Thus the polynomial $F\left(X^{2}, Y^{2}, Z^{2}\right)$ is even in $X, Y, Z$. Note that evenness (or symmetricity) is preserved by the dual curve operation.

Lemma 2.1. Assume that $C=\{F(X, Y, Z)=0\}$ is defined by an even polynomial $F(X, Y, Z)$ in $X$ (respectively symmetric polynomial in $X, Y)$. Then the dual curve $C^{*}$ is defined by an even polynomial $F^{*}\left(X^{*}, Y^{*}, Z^{*}\right)$ in $X^{*}$ (resp. in $\left.X^{*}, Y^{*}\right)$.

Proof. Assume for example that $F(X, Y, Z)$ is even in $X$. Then for any point $P=(X, Y, Z) \in C$, let $P^{\prime}:=(-X, Y, Z)$ is also in $C$. Then it is easy to see that

$$
\operatorname{dual}_{C}\left(P^{\prime}\right)=\left(F_{X}\left(P^{\prime}\right), F_{Y}\left(P^{\prime}\right), F_{Z}\left(P^{\prime}\right)\right)=\left(-F_{X}(P), F_{Y}(P), F_{Z}(P)\right)=\operatorname{dual}_{C}(P)^{\prime}
$$

This implies that $F^{*}\left(X^{*}, Y^{*}, Z^{*}\right)$ is even in $X$. The symmetric case is proved similarly.

Assume that $C \in \mathscr{M}$ is defined by $F(X, Y, Z)=0$. If $F$ is an even polynomial in the variable $X$ (respectively a symmetric polynomial in $X, Y$ ), then 6 cusps are stable by the involution $(X, Y, Z) \mapsto(-X, Y, Z)$ (resp. by $(X, Y, Z) \mapsto$ 
$(Y, X, Z))$. Then there exists a homogeneous polynomial $F_{2}(X, Y, Z)$ of degree 2 which is even in $X$ (resp. symmetric in $X, Y$ ) such that the conic $F_{2}(X, Y, Z)=$ 0 passes through the 6 cusps of $C$. By the criterion of Degtyarev [D], the sextic $F(X, Y, Z)=0$ is of a torus type.

Now we take a generic $C \in \mathscr{M}^{n m l}$ and consider the dual curve $\psi(C)^{*}$ and let $\tilde{G}\left(X^{*}, Y^{*}, Z^{*}\right)$ be a defining homogeneous polynomial of degree 12 , where $\left(X^{*}, Y^{*}, Z^{*}\right)$ is the dual coordinates of $(X, Y, Z)$. As $\tilde{F}(X, Y, Z)$ is even in $X, Y, Z$, so is $\tilde{G}\left(X^{*}, Y^{*}, Z^{*}\right)$ in $X, Y, Z$ by Lemma 2.1 .

Proposition 2.2. $\psi(C)^{*}$ has 4 nodes on each coordinate axis $X^{*}=0, Y^{*}=$ 0 or $Z^{*}=0$.

Proof. Let $C=\{F(X, Y, Z)=0\}$ and let us consider the discriminant polynomial $\Delta_{Y} F$ with respect to $Y$-variable. This is a homogeneous polynomial of degree 30 in $X, Z([\mathrm{O} 2])$. We assume that the singularities of the sextic $F(X, Y, Z)=0$ are not on the coordinate axis. Assume that $P:=(\alpha, \beta, \gamma) \in C$ is a singular point of $C$ with Milnor number $\mu$ and multiplicity $m$. Then $\Delta_{Y} F(X, Z)$ has a linear term $(\gamma X-\alpha Z)^{\rho}$ with $\rho \geq \mu+m-1$ and the equality holds if the line $\gamma Y-\beta Z=0$ is generic with respect to $C$ (see [O3]). Thus to each cusp (respectively to each node), there is an associated linear term with multiplicity 3 (resp. with multiplicity 2). The factor $X=0$ and $Z=0$ has also multiplicity 2 in $\Delta_{Y} F(X, Z)=0$, as they are bi-tangent lines. Assume $C$ is generic in $\mathscr{M}$. Then the sum of degrees is $18+6+4=28$ by the above consideration. Thus there exists two simple tangent lines of the form $X-\eta_{1} Z=0$ and $X-\eta_{2} Z=0$ for some $\eta_{1}, \eta_{2} \neq 0$. Then four lines $X= \pm \sqrt{\eta_{i}} Z, i=1,2$ are bi-tangent lines for the curve $\psi(C)$. This implies that $\left(1,0, \pm \sqrt{\eta_{i}}\right), i=1,2$ are nodes of the dual curve $\psi(C)^{*}$. Thus the coordinate axis $Y^{*}=0$ contains 4 nodes of $\psi(C)^{*}$. By the same argument, $X^{*}=0$ and $Z^{*}=0$ contains also 4 nodes respectively. The non-emptiness of "generic" curves in $\mathscr{M}$ in the above sense is not obvious but it follows from the example below.

Definition 2.3. For $C \in \mathscr{M}^{n m l}$, we define a polynomial of degree 6 by $G\left(X^{*}, Y^{*}, Z^{*}\right):=\tilde{G}\left(\sqrt{X^{*}}, \sqrt{Y^{*}}, \sqrt{Z^{*}}\right)$ and we define $l(C)$ by the sextics defined by $G\left(X^{*}, Y^{*}, Z^{*}\right)=0$. For $C \in \mathscr{M}$, take $g \in G$ so that $C^{g} \in \mathscr{M}^{n m l}$ and we define an involution $\bar{l}: \mathscr{M} / G \rightarrow \mathscr{M} / G$ by $\bar{l}(C)=\imath\left(C^{g}\right)$.

Claim 1. $\bar{l}(C) \in \mathscr{M}$ for a generic $C \in \mathscr{M}$ and $\bar{l}$ is an involution which preserves the type of sextics, that is, we have the commutative diagram:

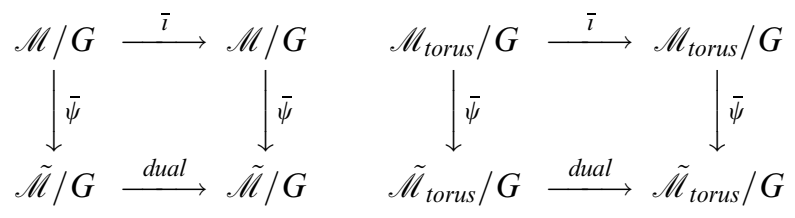


Proof. We may assume that $C \in \mathscr{M}^{n m l}$. By the above consideration, we have seen that the dual curve $\psi(C)^{*}$ of $\psi(C)$ is defined by a polynomial $G\left(X^{*}, Y^{*}, Z^{*}\right)$ of degree 12 which is even in each of the three variables and it has 24 cusps and 12 nodes outside of coordinate axis and 4 nodes on each coordinate axis. Thus $\imath(C)$ has 6 cusps and 3 nodes. Note that nodes of $\psi(C)^{*}$ on the coordinate axes are mapped on simple tangents on the corresponding coordinate axes of $l(C)$. Thus the curve $l(C)$, defined by $g\left(\sqrt{x^{*}}, \sqrt{y^{*}}\right)=0$, belongs to $\mathscr{M}^{n m l}$. Finally we will show that $l$ keeps the type of the curve. As the curves $\left\{\bar{l}(C) ; C \in \mathscr{M}_{\text {torus }} / G\right\}$ are topologically equivalent, the image is contained in a connected component. Thus it is enough to show that there exists a $C \in$ $\mathscr{M}_{\text {torus }} / G$ such that $\bar{l}(C) \in \mathscr{M}_{\text {torus }} / G$. To see this, it is enough to take $C \in \mathscr{M}_{\text {torus }}^{\text {nml }}$ whose defining polynomial $F(X, Y, Z)$ is symmetric in each of $X, Y$. Then $\tilde{F}(X, Y, Z)$ is also symmetric in $X, Y$. This implies also that $\tilde{G}\left(X^{*}, Y^{*}, Z^{*}\right)$ and $G\left(X^{*}, Y^{*}, Z^{*}\right)$ symmetric in $X^{*}, Y^{*}$. By Degtyarev's criterion, this implies that $l(C)$ is a sextic of a torus type.

Thus we have proved the following:

THEOREM 2.4. There exists an involution $\bar{\imath}$ on the quotient moduli space $\mathscr{M} / G$ which is defined on generic points such that $\bar{l}$ is different from the dual curve operation and $\bar{l}$ preserves the types of the sextics, that is $\bar{\imath}(C) \in \mathscr{M}_{\text {torus }} / G \Leftrightarrow C \in$ $\mathscr{M}_{\text {torus }} / G$.

The following example shows that $\bar{l}(C) \neq C^{*}$ in general.

Example 2.5. Let $C \in \mathscr{M}_{\text {torus }}^{\text {nml }}$ be the sextic defined by the symmetric polynomial:

$$
f:=-684\left(x^{3} y+x y^{3}\right)-1055\left(x^{3}+y^{3}\right)+2235\left(x^{2}+y^{2}\right)-2178(x+y)+
$$
$(819 / 16)\left(x^{5} y+y^{5} x\right)+(1767 / 16)\left(x^{4} y^{2}+x^{2} y^{4}\right)+(881 / 8) y^{3} x^{3}+(405 / 16)\left(x^{6}+y^{6}\right)$ $-(873 / 8)\left(x^{5}+y^{5}\right)+(2001 / 4)\left(x^{4}+y^{4}\right)-(971 / 8)\left(x^{4} y+x y^{4}\right)-(6947 / 2) y^{2} x^{2}+$ $2268+1038\left(x^{2} y+x y^{2}\right)-4883 y x-(375 / 2)\left(x^{2} y^{3}+x^{3} y^{2}\right)$.

Then $\psi(C)$ is defined by $f\left(x^{2}, y^{2}\right)$ and $\psi(C)^{*}$ is defined by $g\left(x^{* 2}, y^{* 2}\right)=0$ and $t(C)$ is the sextic defined by the symmetric polynomial

$$
g\left(x^{*}, y^{*}\right):=908294 x^{* 2} y^{* 2}-354000\left(x^{*} y^{* 2}+x^{* 2} y^{*}\right)+302745\left(y^{* 4}+x^{* 4}\right)+
$$
529284 $\left(x^{* 4} y^{* 2}+y^{* 4} x^{* 2}\right)-396458\left(x^{*} y^{* 4}+y^{*} x^{* 4}\right)-722148\left(x^{* 3} y^{* 2}+y^{* 3} x^{* 2}\right)+$ $11340\left(y^{* 6}+x^{* 6}\right)-109170\left(x^{* 5}+y^{* 5}\right)+86296 x^{*} y^{*}+482724\left(x^{* 3} y^{*}+y^{* 3} x^{*}\right)-$ $158508\left(y^{*} x^{* 5}+y^{* 5} x^{*}\right)+103096 y^{* 3} x^{* 3}-22230\left(x^{*}+y^{*}\right)-203920\left(y^{* 3}+x^{* 3}\right)+$ $90570\left(y^{* 2}+x^{* 2}\right)+2025$

The dual curve $C^{*}$ of $C$ is defined by the following symmetric polynomial and we can easily check that $\bar{l}(C) \neq C^{*}$ in $\mathscr{M} / G$.

$$
h\left(x^{*}, y^{*}\right):=3\left(x^{* 4}+y^{* 4}\right)+14\left(x^{* 3}+y^{* 3}\right)+3\left(x^{* 2}+y^{* 2}\right)+4\left(y^{*} x^{* 4}+x^{*} y^{* 4}\right)+
$$
$36\left(y^{*} x^{* 3}+x^{*} y^{* 3}\right)+6\left(y^{*} x^{* 2}+x^{*} y^{* 2}\right)-2 y^{*} x^{*}+12\left(y^{* 2} x^{* 4}+x^{* 2} y^{* 4}\right)+84\left(y^{* 2} x^{* 3}+\right.$ $\left.x^{* 2} y^{* 3}\right)+14 y^{* 2} x^{* 2}+88 y^{* 3} x^{* 3}$ 
Proof. Put $C^{\prime}:=\imath(C)$. We can see that $C^{*}$ and $C^{\prime}$ are not in the same orbit of $\operatorname{PSL}(3 ; C)$. In fact, assume that there exists a $A \in \operatorname{PSL}(3 ; C)$ such that $\left(C^{*}\right)^{A}=C^{\prime}$. Then $A$ maps nodes to nodes. This implies that $A$ permutes the three points $(0,0,1),(0,1,0)$, and $(1,0,0)$. Thus $A$ is a scalar multiplication of the coordinates $\left(X^{*}, Y^{*}, Z^{*}\right) \mapsto\left(\alpha X^{*}, \beta Y^{*}, \gamma Z^{*}\right)$, followed by a persutaion $\sigma \in \mathfrak{S}_{3}$. These actions does not change the number of monomials in $x^{*}$ and $y^{*}$. Thus $h^{A}=g$ is impossible as $g$ has 28 monomials while $h$ has only 19 monomials.

Remark. We know that $\mathscr{M}_{\text {torus }} / G$ is irreducible of dimension 4 , in which one dimensional subvariety comes as the image of Gauss map (= dual curves) of 3 (3,4)-cuspidal sextics of torus type $([\mathrm{O} 4])$. We do not know either the irreduciblity of $\mathscr{M}_{\text {gen }} / G$ or the dimension. Only thing we know is that it contains one dimensional subvariety coming from $3(3,4)$-cuspidal non-torus sextics as the image of Gauss map. However any such curve $C$ is special in the sense $C^{*}$ is not contained in $\mathscr{M}_{\text {gen }} / G$. We do not have any explicit example of a generic element $C \in \mathscr{M}_{\text {gen }} / G$ which has three bitangent lines.

\section{REFERENCES}

[B-K] E. Brieskorn and H. KNÖRRER, Ebene Algebraische Kurven, Birkhäuser, Basel, 1981.

[D] A. Degtyarev, Alexander polynomial of a curve of degree six, J. Knot Theory and its Ramification, 3 (1994), 439-454.

[N] M. Namba, Geometry of Projective Algebraic Curves, Dekker, New York, 1984.

[O1] M. OKa, Symmetric plane curves with nodes and cusps, J. Math. Soc. Japan, 44 (1992), 375-414.

[O2] М. Ока, Flex curves and their applications, Geom. Dedicata, 75 (1999), 67-100.

[O3] M. OKA, Geometry of cuspidal sextics and their dual curves, to appear in Singularities and Arrangements, Sapporo-Tokyo 1998, Kinokuniya.

[O4] M. OKA, Elliptic curves from sextics, preprint, 2000.

[W] R. WaLker, Algebraic Curves, Dover, New York, 1949.

Department of Mathematics

TOKyo Metropolitan University

Minami-OHSAWA, HaChioJi-SHI

TOKYO, 192-0397, JAPAN

E-mail: oka@comp.metro-u.ac.jp 